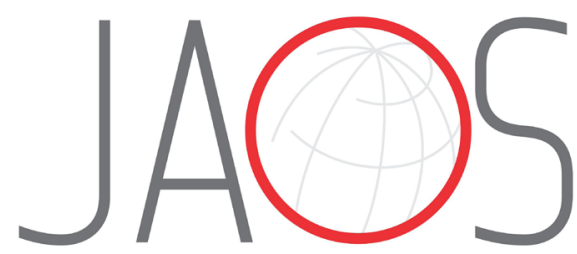

\title{
miR-9-1 gene methylation and DNMT3B (rs2424913) polymorphism may contribute to periodontitis
}

\section{Abstract}

Marina Castro COÊLHO${ }^{1} \#$ iD Ingrid Costa QUEIROZ#\# ID José Maria Chagas VIANA FILHO ${ }^{1}$ iD

Sabrina Garcia de AQUINO ${ }^{1,3}$ iD

Darlene Camati PERSUHN ${ }^{4}$ Naila Francis Paulo de OLIVEIRA ${ }^{1,4}$ iD
Genetic and epigenetic changes have been associated with periodontitis in various genes; however, little is known about genes involved in epigenetic mechanisms and in oxidative stress. Objective: This study aims to investigate the association of polymorphisms C677T in MTHFR (rs1801133) and -149C $\rightarrow T$ in DNMT3B (rs2424913), as well as the methylation profiles of MTHFR, miR-9-1, miR-9-3, SOD1, and CAT with periodontitis. The association between polymorphisms and DNA methylation profiles was also analyzed. Methodology: The population studied was composed of 100 nonsmokers of both sexes, divided into healthy and periodontitis groups. Genomic DNA was extracted from the epithelial buccal cells, which were collected through a mouthwash. Polymorphism analysis was performed through polymerase chain reaction-restriction fragment length polymorphism (PCR-RFLP), while methylation-specific PCR (MSP) or combined bisulfite restriction analysis techniques were applied for methylation analysis. Results: For DNMT3B, the $T$ allele and the $\Pi$ genotype were detected more frequently in the periodontitis group, as well as the methylated profile on the miR-9-1 promoter region. There was also a tendency towards promoter region methylation on the CAT sequence of individuals with periodontal disease. Conclusion: The polymorphism -149C $\rightarrow$ T in DNMT3B (rs2424913) and the methylated profile of the miR-9-1 promoter region are associated with periodontitis.

Keywords: Periodontitis. Epigenetic. DNA methylation. Polymorphism. Oxidative stress. MicroRNA.
Submitted: September 30, 2019 Modification: November 11, 2019

Accepted: November 27, 2019
Dra. Naila Francis Paulo de Oliveira Universidade Federal da Paraíba - Centro de Ciências Exatas e Naturais - Departamento de Biologia Molecular - Cidade Universitária Campus I - 58051-900 - João Pessoa - PB - Brasil. Phone: +55 83 3216-7643 e-mail: nailafpo@dbm.ufpb.br
${ }^{1} U$ niversidade Federal da Paraíba, Centro de Ciências da Saúde, Programa de Pós Graduação em Odontologia, João Pessoa, Paraíba, Brasil.

¿Universidade Federal da Paraíba, Centro de Ciências da Saúde, João Pessoa, Paraíba, Brasil. ${ }^{3}$ Universidade Federal da Paraíba, Centro de Ciências da Saúde, Departamento de Odontologia Clínica e Social, João Pessoa, Paraíba, Brasil.

${ }^{4}$ Universidade Federal da Paraíba, Centro de Ciências Exatas e da Natureza, Departamento de Biologia Molecular, João Pessoa, Paraíba, Brasil. 


\section{Introduction}

Periodontitis is defined as a chronic, inflammatory, and multifactorial disease; it is associated with a dysbiotic biofilm and characterized by the progressive destruction of the dental insertion apparatus. ${ }^{1}$ Epidemiological studies revealed that most adult individuals are affected by mild and moderate forms of this disease, while $5-20 \%$ of any population has severe periodontitis. In Brazil, the periodontitis prevalence is higher than the global average. ${ }^{2}$ If not treated, periodontitis is a risk factor for prominent noncommunicable diseases, including pneumonia, gastritis, diabetes mellitus, and chronic renal disorders; also, the number of lost teeth is predictive of mortality by cardiovascular conditions. ${ }^{3}$

As a multifactorial disease, genetic and environmental aspects are important factors that lead to the susceptibility to periodontitis. Environmental factors may alter the cellular epigenome, which may affect gene expression. The epigenome refers to chemical modifications that may occur on DNA and histones, and to the individual's non-coding RNA profile. ${ }^{4}$ Epigenetic marks comprise DNA methylation, chemical alterations on histones (methylation, acetylation, and phosphorylation), and small, noncoding RNA (microRNA).

DNA methylation occurs in the CpG dinucleotides, mainly in gene promoter regions, and controls expression at the transcriptional level by hindering the access of transcription factors to their binding sites. Methyl radicals are added to the DNA by DNA methyltransferases (DNMT), and these enzymes use the $S$-adenosyl methionine (SAM) radical as substrate. The SAM molecules are synthesized during the folate metabolic pathway by the methylenetetrahydrofolate reductase (MTHFR) activity. MicroRNAs, in turn, control the gene expression at the post-transcriptional level by biding to target messenger RNA (mRNA) and inhibiting their translation. Approximately $50 \%$ of the microRNA genes are associated with CpG islands, and their expression may be regulated by DNA methylation pathways. $^{5}$

Genetic polymorphisms in MTHFR and DNMT may influence the DNA methylation profile; as mentioned, these enzymes originate the SAM radical and use it as substrate for DNA methylation, respectively. The polymorphism C677T MTHFR (rs1801133) reduces enzymatic activity, resulting in a decrease in SAM concentration. The polymorphism $-149 \mathrm{C} \rightarrow \mathrm{T}$ DNMT3B (rs2424913), on the other hand, enhances the transcription of the sequence. ${ }^{6}$ In fact, some studies demonstrated that rs1801133 is associated with global and site-specific hypomethylation, ${ }^{7}$ while rs 2424913 is associated with hypermethylation of specific genes. $\underline{8}$

DNA methylation profile alterations have been described in periodontitis, especially in Toll-like receptor, cytokine, and metalloproteinase genes. ${ }^{9,10}$ Notably, some of these studies reported epigenetic alterations outside of gingival tissue, such as buccal epithelium and blood. This phenomenon may suggest these changes are not limited to the gingiva and, at the same time, reflect the cellular condition. Information on the methylation profile of microRNA genes (miR) and on the genes involved in the oxidative stress pathway are still scarce. However, the microRNA expression as well as antioxidant proteins, such as superoxide dismutase and catalase, are altered in periodontitis patients' gingival tissue cells and saliva. ${ }^{11,12}$

It speculated that genetic polymorphisms in DNA methylation pathway genes (MTHFR and DNMT) and methylation profile alterations in microRNA genes and in genes from the oxidative stress pathway may be involved in periodontitis pathogenesis. This study focused on these genes because, as mentioned earlier, MTHFR and DNMT are involved in DNA methylation processes and both polymorphisms and methylation of these genes are involved in inflammatory diseases, ${ }^{13-15}$ probably affecting the levels of products originated during the folate metabolic cycle, such as homocysteine and SAM radicals. ${ }^{7}$ Genes related to oxidative stress pathways, such as SOD and CAT, have been associated with periodontitis, ${ }^{12}$ while microRNA genes are involved in inflammatory diseases by silencing several mRNA targets related to cellular processes, including cellular homeostasis, proliferation, differentiation, development, growth, and apoptosis, ${ }^{11}$ particularly the miR-9 family targets transcripts of inflammatory cytokines. ${ }^{16,17}$ However, to the best of our knowledge, nothing is known, in the context of periodontitis, about genetic and epigenetic marks in the above genes.

Therefore, the main objective of this study was to investigate the association between the MTHFR rs1801133 and the DNMT3B rs2424913 polymorphisms, and methylation profiles from MTHFR, miR-9-1, miR-9-3, superoxide dismutase (SOD1), and catalase (CAT) genes with periodontitis. The study also assessed the association between these 
polymorphisms, the DNA methylation profiles, and periodontitis.

\section{Methodology}

\section{Studied population and research ethics}

The study was conducted according to the Declaration of Helsinki and was approved by the Research Ethics Committee (CAAE: 64578717.4.0000.5188). A convenience sample of unrelated nonsmoking subjects (30-70 years old) was recruited for this study from the patient pool of the Periodontics Clinic (Department of Clinical and Social Odontology) from May 2017 to November 2018. The studied population from the Northeast region of Brazil comprised 100 individuals of both sexes who were generally healthy; their diagnosis was based on the American Academy of Periodontology guidelines. ${ }^{18}$ The medical and dental history were recorded including the measurements of probing depth (PD), bleeding on probing, and clinical attachment level (CAL) at six sites per tooth. Following the diagnosis, the participants were divided into two groups according to the coauthor's supervision: the control group $(n=50)$, which included individuals with no clinical signs of gingival inflammation, with CAL and $\mathrm{PD} \leq 3 \mathrm{~mm}$; and the chronic periodontitis group $(n=50)$, comprising patients with at least 3 teeth presenting CAL and $P D \geq 5 \mathrm{~mm}$ and clinical signs of inflammation. Exclusion criteria for this study included smoking, diabetes, history of periodontal treatment, pregnancy, hepatitis, HIV infection, and use of antiinflammatory medication in the last 6 months.

\section{Sample collection and DNA extraction}

Buccal mucosa cells were collected from a oneminute mouthwash with $6 \mathrm{~mL}$ of autoclaved dextrose ( $3 \%)$, and after the collection $3 \mathrm{~mL}$ of TNE buffer was added. The samples were taken to the Laboratory of Molecular Genetics (Department of Molecular Biology), where nucleic acid extraction and molecular analysis were performed. Subsequently, the samples were centrifuged at 3,000 rpm for $10 \mathrm{~min}$ and the supernatant was discarded. Lysis solution was added to the pelleted epithelial cells and the samples were stored at $-20^{\circ} \mathrm{C}$ until DNA extraction. The genomic DNA was purified with $8 \mathrm{M}$ ammonium acetate. ${ }^{19}$ The amount and the purity of the isolated DNA were measured with a Nanodrop spectrophotometer using the OD 260/280 ratio. The sample was considered pure when the mean value between two readings was 1.8 or greater.

\section{Genetic polymorphism analysis of MTHFR and DNMT3B}

The analysis of the single nucleotide polymorphisms (SNPs) C677T MTHFR (rs1801133) and $-149 \mathrm{C} \rightarrow \mathrm{T}$ DNMT3B (rs2424913) was performed through polymerase chain reaction-restriction fragment length polymorphism (PCR-RFLP), where DNA fragments are amplified by PCR and digested by a restriction enzyme. The presence or absence of the SNP was obtained according to the restriction enzyme activity. The 15$\mu \mathrm{L}$ reactions contained $7.5 \mu \mathrm{L}$ GoTaq $^{\circledR} \mathrm{G} 2$ Hot Start Green Master Mix (Promega), $1 \mu \mathrm{L}$ of each primer (10 $\mu \mathrm{M}), 1 \mu \mathrm{L}$ of DNA, and nuclease-free water. PCR and enzymatic digestion conditions, as well as the chosen primers, were previously described. ${ }^{15,16,20,21}$ Genotypes were analyzed through vertical electrophoresis in $6 \%$ polyacrylamide gels, followed by coloring with silver nitrate or GelRed ${ }^{\circledR}$ (Biotium). The $677 \mathrm{CC} / \mathrm{CT} / \mathrm{TT}$ and the $-149 \mathrm{CC} / \mathrm{CT} / \mathrm{TT}$ genotypes were identified by their band pattern according to the literature. ${ }^{15,16,20,21}$

\section{Methylation analysis of MTHFR, $\operatorname{miR}-9-1$, and miR-9-3 gene promoters}

Analysis of MTHFR, miR-9-1, and miR-9-3 promoter methylation was performed using the methylationspecific PCR (MSP). To perform this technique, the purified DNA was transformed with sodium bisulfite and with hydroquinone for $3 \mathrm{~h}$ at $70^{\circ} \mathrm{C}$ for complete unmethylated cytosine conversion into uracil. The differences in the DNA sequences after the bisulfite treatment were detected by amplification with specific primers for methylated and non-methylated sequences, as previously described. The PCR conditions were also previously described. ${ }^{22,23}$ After amplification, the methylation profiles were visualized through vertical electrophoresis of $7 \mu \mathrm{l}$ of amplified DNA in $6 \%$ polyacrylamide gels, followed by silver nitrate coloring. The profiles were categorized as methylated, with amplification for the methylated condition only; unmethylated, with amplification for the nonmethylated condition; and partially methylated, when amplification was observed for both conditions. 
Methylation analysis of SOD1 and CAT gene promoters

SOD1 and CAT promoter methylation analysis was performed using combined bisulfite restriction analysis (COBRA), in which the DNA is submitted to a bisulfite treatment followed by a PCR amplification, and enzymatic digestion with restriction enzymes specific for transformed $(\mathrm{C} \rightarrow \mathrm{U}$ ) or conserved $\mathrm{CpG}$ sites. ${ }^{24}$ The DNA was transformed as previously mentioned. The primer sequences for each gene (CAT: F:5'-GTTTTAATTGTTGAGTAATAAATGAGA-3' and R:5'-AAAAAAAACAACCTTCTITCA-3', 199 base pairs (bp); SOD1: F:5'-GGTIITTAATTGTTGGGTTAGAG-3' and R:5'- ACTCAACCAATCAACACCAC-3', 175 bp) were obtained using the Methyl Primer Express v1.0 (Applied Biosystems) software according to the promoter region sequence entered into the Genome Browser (chr21:31,659,622-31,668,931 and chr11:34,438,925-34,472,062, respectively). Fifteen $\mu \mathrm{L}$ reactions contained 7.5 $\mu \mathrm{L}$ GoTaq ${ }^{\circledR}$ Hot Start Green Master Mix (1X; Promega), $2 \mu \mathrm{L}$ of the primer sets $(10 \mu \mathrm{M}), 5 \mu \mathrm{L}(\sim 100 \mathrm{ng})$ of transformed DNA, and nuclease-free water. Forty-cycle amplifications were performed for both sequences. For $C A T$, the annealing temperature was $55^{\circ} \mathrm{C}$, while for $S O D$ it was $51^{\circ} \mathrm{C}$.

After amplification, $3 \mu \mathrm{L}$ of the PCR products were digested by the restriction enzyme AciI (GCGG; Thermo Fisher) for $3 \mathrm{~h}$ at $37^{\circ} \mathrm{C}$, followed by vertical electrophoresis in a $6 \%$ polyacrylamide gel, and coloring with GelRed ${ }^{\circledR}$. Methylation profiles were categorized as methylated or with enzymatic digestion when the GCGG sites were not transformed during treatment; unmethylated or without enzymatic digestion when the GCGG sites were transformed during the treatment; and partially methylated or with partial digestion. To ensure specificity of all methylation protocols, completely methylated and demethylated DNA controls were used (Cells-to-CpG ${ }^{\mathrm{TM}}$ Methylated \& Unmethylated gDNA Control Kit, Life Technologies).

\section{Statistical analysis}

All data were compiled in an Excel ${ }^{\circledR}$ spreadsheet. Descriptive statistics was used for demographic data. The Hardy-Weinberg equilibrium (HWE) was calculated for each polymorphism using the chi-squared test. The Fisher's Exact or the chi-squared tests were also used for the analysis of possible associations between genotypic frequencies, allelic frequencies, methylation profiles, and the outcome (periodontitis), as well as the association between the methylation profiles and the presence of polymorphisms. All analyses were performed using the BioEstat 5.3 software. p-values $<0.05$ were considered statistically significant.

\section{Results}

Demographic data are shown in Table 1.

\section{Genetic polymorphism analysis}

Table 1 shows the genotypic and allelic distributions in the population studied. Both groups are in accordance with the HWE for each of the polymorphisms studied (DNMT3B (rs2424913): control, $\mathrm{p}=0.35$ and periodontitis, $\mathrm{p}=0.78$; MTHFR (rs1801133): control, $\mathrm{p}=0.31$ and periodontitis, $\mathrm{p}=0.16)$. For $D N M T 3 B$, the $T$ allele was more frequent in the periodontitis (52\%) compared with the control group ( $33 \% ; p=0.01)$. The same trend was observed for the $\Pi$ genotype, which was more frequent in the periodontitis (28\%) compared with the control group ( $8 \% ; p=0.01)$. Individuals with the $\Pi$ genotype are approximately four times more likely to develop periodontitis [OR: $4.47(1.35 ; 14.75)]$. For MTHFR, the C allele and the genotypes $\mathrm{CC}$ and $\mathrm{CT}$ were the most common in both groups; therefore, no significant differences were observed between the groups (Table 1 and Figure 1 ).

\section{Methylation profile analysis}

For MTHFR, the unmethylated profile appeared more frequently in both groups $(92.5 \%$ in the control group and $90 \%$ in the periodontitis group). For miR-9-1, methylation occurred in $100 \%$ of the periodontitis patients but only in $77.5 \%$ of the control group participants $(p=0.0024)$. On the other hand, miR-9-3 showed a partially methylated profile in both groups (100\%; Table 1 and Figure 2). For SOD1, the unmethylated profile was the most frequent in both groups ( $85 \%$ of the control group and $79 \%$ of the periodontitis group). For CAT, although no significant difference was observed between the two groups, there was a tendency towards methylation in the periodontitis group, since the methylated profile was more common amongst these patients ( $55 \%=50 \%$ partially methylated $+5 \%$ methylated $)$ when compared with the control group $(42.5 \%=40 \%$ partially methylated $+2.5 \%$ methylated; $p=0.077$; 
Table 1 and Figure 3). The DNMT3B TT genotype and the miR-9-1 methylation profile were considered for statistical association analysis, because both genes showed a statistically significant difference between the two groups. However, no association between these variables and periodontitis was found.

Table 1- Demographic, polymorphism, and DNA methylation data

\begin{tabular}{|c|c|c|c|}
\hline Demography & $\begin{array}{c}\text { Control } \\
(n=50)\end{array}$ & Periodontitis $(n=50)$ & $P$ value \\
\hline Age (years) & $38.7( \pm 7.5)$ & $49.0( \pm 11.3)$ & 0.0001 \\
\hline$\%$ men & 12 & 22 & 0.001 \\
\hline$\%$ women & 38 & 28 & \\
\hline Probing depth (mm) & $\leq 3$ & $\geq 5$ & \\
\hline Polymorphism & $n=50$ & $n=50$ & \\
\hline \multicolumn{4}{|l|}{ DNMT3B genotype } \\
\hline CC & $21(42 \%)$ & $12(24 \%)$ & $0.01^{*}$ \\
\hline CT & $25(50 \%)$ & $24(48 \%)$ & OR:4.47; \\
\hline TT & $04(08 \%)$ & $14(28 \%)$ & {$[1.35 ; 14.75]$} \\
\hline \multicolumn{4}{|l|}{ Allele frequency $\%$} \\
\hline C & 67 & 48 & $0.01^{*}$ \\
\hline $\mathrm{T}$ & 33 & 52 & OR: 2.19; \\
\hline \multicolumn{4}{|l|}{ MTHFR genotype } \\
\hline $\mathrm{CC}$ & $26(52 \%)$ & $21(42 \%)$ & \\
\hline СT & $18(36 \%)$ & $26(52 \%)$ & $>0.05$ \\
\hline TT & $06(12 \%)$ & $03(06 \%)$ & \\
\hline \multicolumn{4}{|l|}{ Allelle frequency $\%$} \\
\hline C & 70 & 68 & $>0.05$ \\
\hline $\mathrm{T}$ & 30 & 32 & \\
\hline \multicolumn{4}{|l|}{ DNA methylation } \\
\hline MTHFR & $n=40$ & $n=40$ & \\
\hline M & $01(2.5 \%)$ & $0(0 \%)$ & \\
\hline U & $37(92.5 \%)$ & $36(90 \%)$ & $>0.05$ \\
\hline$M$ and $U$ & $02(5 \%)$ & $04(10 \%)$ & \\
\hline miR-9-1 & $n=40$ & $n=40$ & \\
\hline $\mathrm{M}$ and $\mathrm{U}$ & $31(77.5 \%)$ & $40(100 \%)$ & $0.0024 \#$ \\
\hline U & $09(22.5 \%)$ & 0 & RR:1.29; [1.09;1.52] \\
\hline \multicolumn{4}{|l|}{ miR-9-3 } \\
\hline$M$ and $U$ & $40(100 \%)$ & $40(100 \%)$ & $>0.05$ \\
\hline U & 0 & 0 & \\
\hline SOD1 & $n=40$ & $n=38$ & \\
\hline M & 0 & 0 & \\
\hline U & $34(85 \%)$ & 30 (79\%) & $>0.05$ \\
\hline$M$ and $U$ & $06(15 \%)$ & $08(21 \%)$ & \\
\hline CAT & $n=40$ & $n=40$ & \\
\hline M & $01(2.5 \%)$ & $02(5 \%)$ & \\
\hline U & $23(57.5 \%)$ & $18(45 \%)$ & $0.077 \#$ \\
\hline $\mathrm{M}$ and $\mathrm{U}$ & $16(40 \%)$ & $20(50 \%)$ & \\
\hline
\end{tabular}

\section{Periodontitis has been associated with genetic polymorphisms in several cellular pathways. ${ }^{25}$ From 2009 onwards, ${ }^{9}$ methylation analysis studies in the context of periodontal disease have markedly increased; however, many genes still need to be \\ Discussion} (n) was found

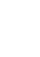

$M=$ methylated, $U=$ unmethylated, $M$ and $U=$ partially methylated * Fisher's exact, \#x2 Test. 

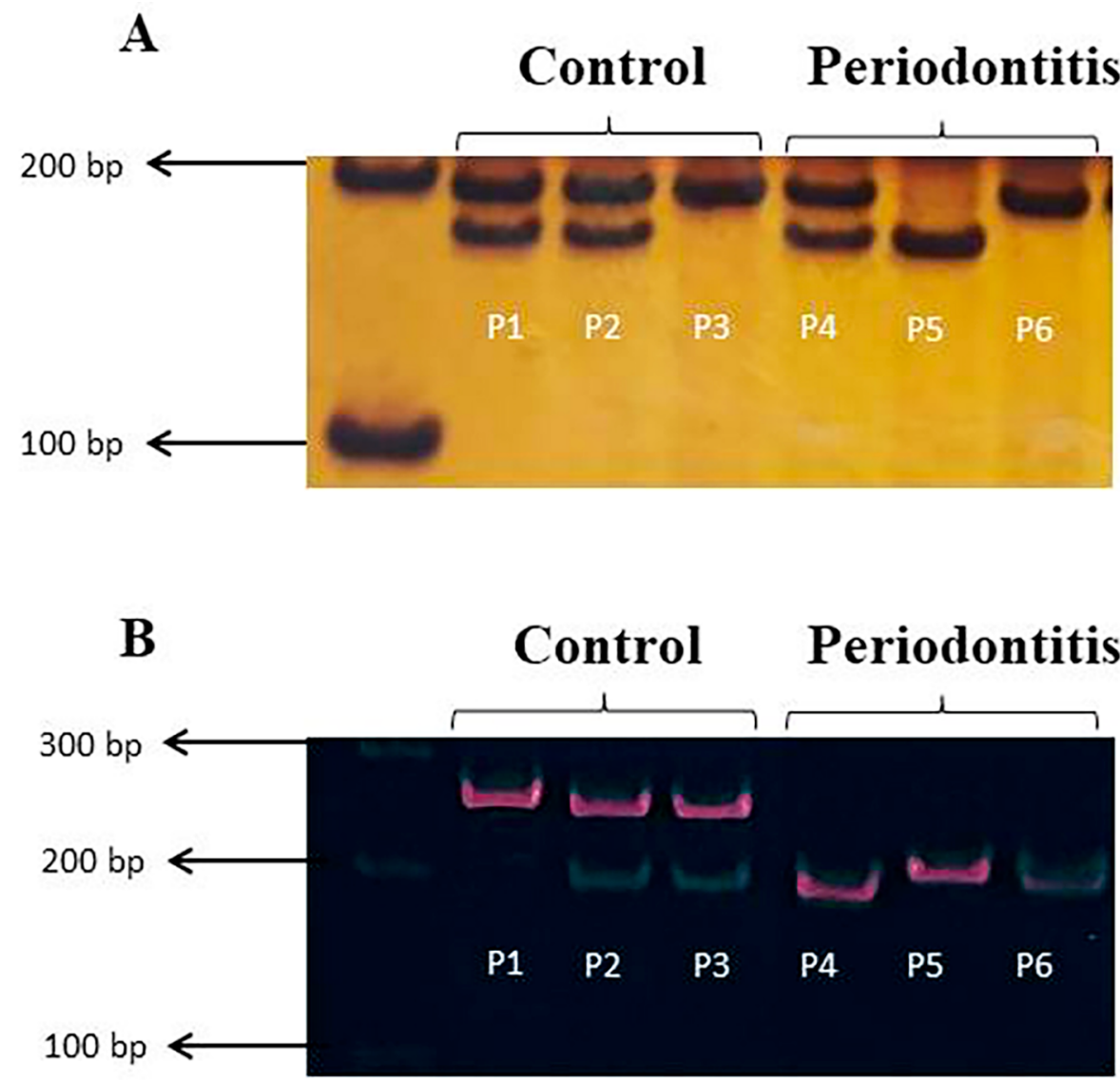

Figure 1- Representative bands of MTHFR and DNMT3B stained with silver nitrate or GelRed ${ }^{\circledast}$ following the PCR-RFLP method for both control and periodontitis groups. (A) Amplicons for the MTHFR SNP in exon: CC=198 bp, CT=198, 175 and 23 bp (not shown), TT=175 and $23 \mathrm{bp}$ (not shown); (B) Amplicons for the DNMT3B SNP in gene promoter: CC=230 bp, CT=230, 172 and 58 bp (not shown), TT=172 and 58 bp (not shown). P1-P6=patient 1-patient 6

analyzed. This study focused on the genes that had not been previously studied in polymorphism or in methylation contexts, and are involved in DNA methylation (MTHFR and DNMT3B), oxidative stress (SOD1 and $C A T$ ), and translation inhibition (noncoding miR-9-1 and miR-9-3).

The population studied showed the $\Pi T$ genotype and the $T$ allele for $D N M T 3 B$ was more frequent in individuals with periodontitis. This gene is part of the DNA methyltransferase gene family, which acts by transferring the methyl radical $\left(\mathrm{CH}_{3}\right)$ to DNA molecules with no previous methylation marks (de novo methylation). The $\mathrm{C} \rightarrow \mathrm{T}$ exchange in the promoter region enhances its own transcription and possibly causes a predisposition to abnormal de novo methylation and to transcription inhibition. ${ }^{26}$ Based on this possibility, the $\Pi T$ genotype could lead to hypermethylation and further silencing of important genes related to the protection against periodontitis. The homozygous TT genotype for the DNMT3B rs2424913 had already been previously associated with head and neck squamous cell carcinoma, ${ }^{21,27}$ while the CT genotype was associated with oral lichen planus and with an increase in expression of the protein encoded by this genotype. ${ }^{15} D N M T 3 B$ has been minimally explored in the periodontitis context and a recent study demonstrated that its expression increases after the administration of Porphyromonas gingivalis in experimental periodontitis. ${ }^{28}$

The MTHFR rs1801133 polymorphism showed no association with periodontitis. The $\mathrm{C}$ allele and the genotypes CC and CT were the most frequent in the entire population studied. The $\mathrm{T}$ allele and the $\Pi$ genotype have been associated with other oral disease contexts, including lichen planus and squamous cell carcinoma. ${ }^{29,30}$ The enzyme encoded by this gene participates in the folate metabolism pathway, which produces the SAM radicals used by the DNMTs as methyl donors for DNA methylation. The $C \rightarrow T$ exchange reduces enzymatic activity and possibly leads to DNA hypomethylation.

In this study, the unmethylated MTHFR profile was recurrent among all participants. A previous study on MTHFR methylation in the buccal mucosa also identified the unmethylated profile as the most frequent amongst both young and elderly individuals. ${ }^{31}$ 
A

Control

Periodontitis

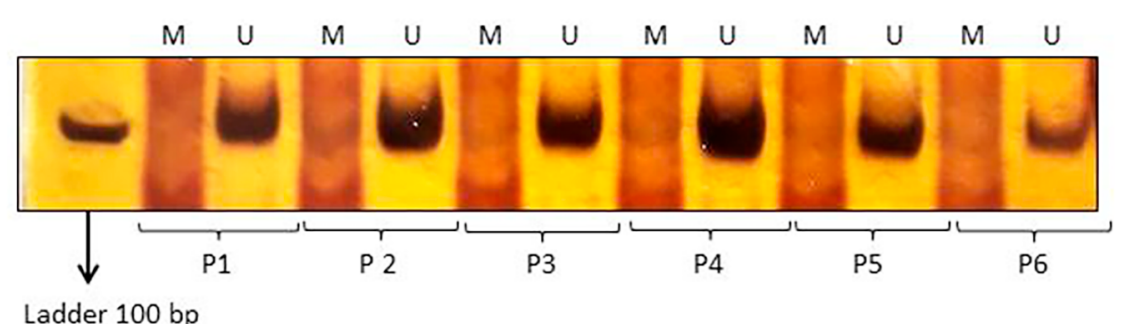

Ladder 100 bp

B

Control Periodontitis
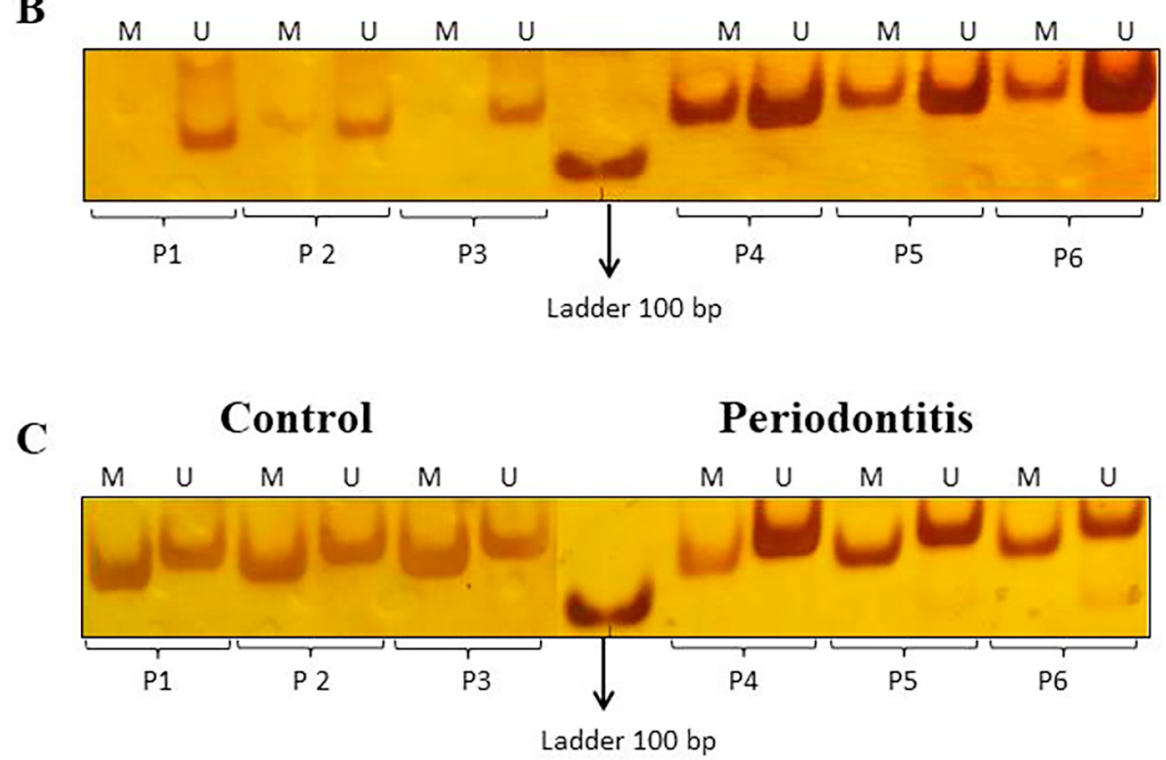

Figure 2- Representative bands of MTHFR and miR-9 stained with silver nitrate following amplification by MSP method for both control and periodontitis groups. (A) Amplicons for methylated and unmethylated (198 bp) status of the MTHFR gene promoter; (B) Amplicons for methylated and unmethylated (110 bp) status of the miR-9-1 gene promoter; (C) Amplicons for methylated and unmethylated (116 bp) status of the miR-9-3 gene promoter. $\mathrm{M}=$ methylated, $\mathrm{U}=$ unmethylated. $\mathrm{P} 1-\mathrm{P} 6=$ patient 1 -patient 6 .

These data collectively demonstrate that this profile is common in buccal epithelial cells and is not associated with oral inflammation. The same CpG site analyzed herein has been studied by our group in patients with diabetic complications; the hypermethylated profile was detected in blood. ${ }^{14}$

The data of this study concerning promoter methylation in microRNA genes revealed that the partially methylated miR-9-3 condition was the standard profile for buccal epithelial cells in individuals with or without periodontitis, while the miR-9-1 methylation was more common in periodontitis patients. According to definitions found in a microRNA database (http://www.mirdb.org), all three loci for miR-9 genes $(1,2$ and 3$)$ in the human genome can be transcribed into the same mature $m i R-9$ sequence.

The miRDB database shows miR-9 may have 991 predictive targets. Hypermethylation of these genes and a decrease in their own expression may increase the levels of such targets, as has been recently shown by Marinho, et al. ${ }^{32}$ (2019). In this study, levels of the rap guanine nucleotide exchange factor, a $m i R$ 9 target, were higher in the periodontitis patients' gingival crevicular fluid. The signaling cascade in which this factor participates may be involved in apoptosis, integrin-mediated signal transduction, and cellular transformation. Another target, the transforming growth factor $\beta-1$ (TGF- $\beta 1$ ) receptor, is also expressed in the gingival tissue of individuals with periodontal disease. ${ }^{16}$ It binds to the anti-inflammatory cytokine TGF- $\beta 1$, which is involved in tissue regeneration and cellular processes such as proliferation, growth, and differentiation.

miR-9-1 hypermethylation has also been observed in cervical and ovarian cancer, and myeloma. ${ }^{17,33,34}$ A study with HeLa cells demonstrated that miR9 hypermethylation reduces its expression and is associated with enhanced interleukin 6 (IL-6) levels, a finding that may indicate that $I L-6$ transcripts are targets for $m i R-9^{17}$. Interleukin 6 levels are higher in 


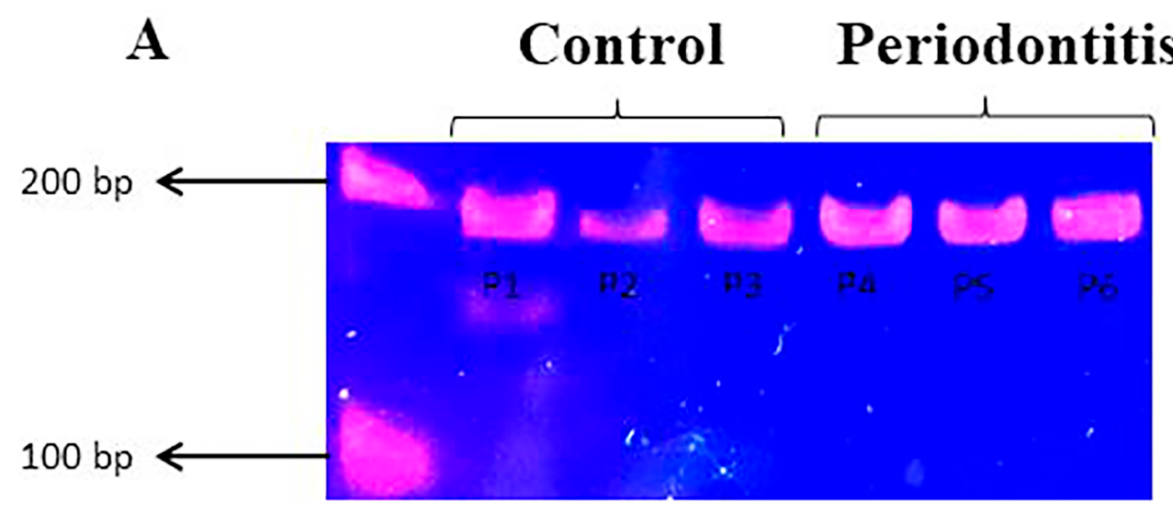

B Control Periodontitis

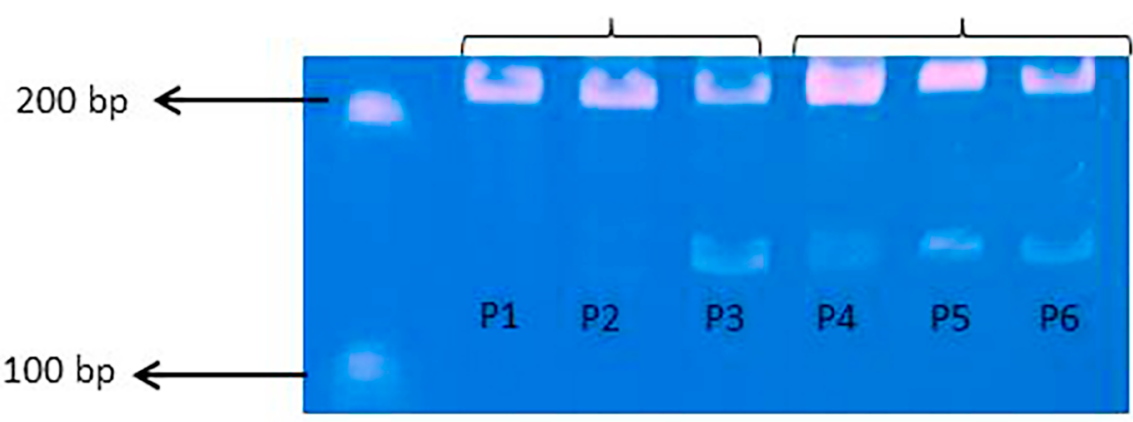

Figure 3- Representative bands stained with Gel Red of SOD1 and CAT following the COBRA method for both control and periodontitis groups. (A) Amplicons after digestion for methylated (139 bp) and unmethylated (175 bp) status of the SOD1 gene promoter; (B) Amplicons after digestion for methylated (167 bp) and unmethylated (199 bp) status of the CAT gene promoter. P1-P6=patient 1-patient 6 .

periodontitis patients when compared with those of control groups. ${ }^{35}$

The partially methylated miR-9-3 condition, in which not all cells present methylation marks in the same CpG sites, had been previously observed for epithelial buccal cells of nonsmoker individuals, reassuring that partial methylation is the common profile for these cells. ${ }^{22}$

Regarding CAT and SOD1 genes, CpG sites were chosen due to their location, because they are immersed in a CpG island near the gene promoter and act as recognition sites for restriction enzymes used in the COBRA technique. Sites -47 for CAT and -184 for SOD 1 have affinity for the Sp1 transcription factor, which positively controls transcription for these genes. This feature may suggest that both sites contribute to regulating each sequence's expression. ${ }^{36,37}$

Catalase and superoxide dismutase, the enzymes that are encoded by $C A T$ and $S O D 1$, respectively, are part of the human enzymatic antioxidant defense group that protects against oxidative damage derived from the reactive oxygen species (ROS). Thus, these enzymes modulate the extension of inflammatory reactions. Both enzyme levels have been evaluated in various contexts and many studies demonstrated a strong association between oxidative stress and periodontal disease. Most researches in this field evaluated transcript levels, protein expression, antioxidant enzymatic activity, total antioxidant capacity, and ROS levels. ${ }^{12}$ Nevertheless, methylation profile studies are scarce, and nothing was known about periodontitis patients.

For SOD1, the unmethylated profile was the most frequent for CpG site -184 in the entire studied population, indicating that this profile is standard for buccal epithelial cells. Similar to this study, an unmethylated profile for various SOD1 promoter region $\mathrm{CpG}$ sites occurs in the cerebral cortex and blood cells in lateral amyotrophic sclerosis patients, as well as in the control individuals. ${ }^{38}$ Although the $S O D 1$ is generally considered to be a maintenance gene due to its ubiquitous and abundant expression, this induction of sequence is finely controlled by complex intracellular events. Alterations in the SOD1 expression have been linked to periodontitis, however, data are still inconclusive, since some studies report increase in the SOD expression/activity while others indicate decline. ${ }^{12,39}$ Considering that gene expression 
may be controlled by DNA methylation, it is important to consider methylation studies on SOD 1 and periodontitis. If the analysis of this study had involved inflammation sites, such as SOD expression studies, some associations could have been detected. In any case, this study infers that the unmethylated profile at site -184 in SOD1 is common for buccal epithelial cells and is not associated with periodontal inflammation.

For CpG site -47 in CAT, there was no significant difference between the groups, but the periodontitis group profile showed a tendency towards methylation. It has been demonstrated that methylated CpG sites near the CAT promoter region are associated with catalase transcription and expression decline in liver tumor cell lines and tumor tissues. ${ }^{40}$ The adverse effects of the CAT promoter methylation upon gene expression have also been reported in studies with hepatic cell lines treated with $\mathrm{CuO}$ nanoparticles. ${ }^{40}$

Catalase level reductions were detected in saliva of individuals with periodontitis when compared to healthy individuals ${ }^{41}$. This decline may be associated with gene methylation, which was a trend in the periodontitis group in this study. Additionally, the CAT hypermethylation observed by Punj, et al. ${ }^{42}$ (2017) occurred via ROS. Periodontal pathogens activate the host's defenses towards ROS formation, which in turn, contributes to the biofilm microorganisms' death. However, prolonged exposure to the ROS may induce a wide range of pathological reactions in the host's tissue, such as membrane lysis, nuclear fragmentation, and enzymatic activation or inactivation. These changes are likely to play a role in the pathogenesis of many inflammatory diseases, from periodontitis to systemic disorders. ${ }^{42}$ Thus, the trend towards methylation demonstrated in this study possibly derives from ROS release of buccal mucosa cells in response to periodontal pathogens. This tendency should be investigated in gingival tissue in addition to ROS and catalase levels analysis.

This study limitations consist of a low sample size for polymorphism studies due to a narrow sample collection period and to well-defined inclusion criteria, and for these reasons, data on the periodontitis group was not subgrouped by disease severity. However, the data of the study referred to the DNMT3B polymorphism rs2424913, which had not been previously studied in the periodontitis context. The finding of this study should be further explored in larger populations with different ethnic backgrounds before any conclusions can be drawn about the role of this gene in periodontitis. Although this study on DNA methylation did not involve the inflammation site, it still showed that profile alterations in buccal mucosa cells, especially in miR-9-1, may be involved in periodontitis.

As a multifactorial disease, periodontitis should be investigated focusing on an individual's genetic and epigenetic aspects. The latter can be modulated by individual habits and even be considered as therapeutic targets. Therefore, the data of this study contribute to the elucidation of molecular mechanisms involved in periodontitis development. Aside from cytokine genes, Toll-like receptors, matrix metalloproteinases, and other molecules mentioned in the literature, it demonstrated that genes involved in DNA methylation and translation inhibition may be involved in periodontitis. Molecular data can be collectively tabulated into a panel of genes indicating susceptibility to periodontal disease and be used as a tool for personalized medicine, contributing to better diagnosis, prognosis, and treatment.

In summary, the conclusion is that the genotype TT DNMT3B (rs2424913) and the methylated profile of the miR-9-1 promoter region are associated with periodontitis. The miR-9-1 promoter methylation yields a relative risk of 1.29 for periodontitis development, while a promoter region polymorphism in the $D N M T 3 B$ causes individuals to be even 4 times more susceptible to periodontal disease.

\section{Acknowledgments}

This study had no specific concession from public, commercial, or nonprofit financing agencies. The authors thank the volunteers who donated buccal mucosa samples and the director of the Periodontics Clinic.

\section{Conflict of interest}

All authors declare no conflict of interest.

\section{Authors' Contribution}

Coêlho, Marina Castro: Data curation (Equal); Formal analysis (Equal); Investigation (Equal); Methodology (Equal); Writing-original draft (Lead); Writing-review \& editing (Lead); Queiroz, Ingrid Costa: Data curation (Equal); Formal analysis (Equal); Investigation (Equal); Methodology (Equal); Writing-original draft (Lead); Writing-review \& editing (Lead); de Oliveira, Naila F. P.: Conceptualization 
(Lead); Investigation (Lead); Methodology (Lead); Project administration (Lead); Supervision (Lead); Writing-original draft (Lead); Writing-review \& editing (Lead); Persuhn, Darlene Camati: Conceptualization (Supporting); Investigation (Supporting); Methodology (Equal); Project administration (Supporting); Writingreview \& editing (Equal); Aquino, Sabrina Garcia: Conceptualization (Supporting); Investigation (Supporting); Methodology (Supporting); Project administration (Supporting); Writing-review \& editing (Equal); Viana Filho, José M. C.: Data curation (Equal); Writing-review \& editing (Supporting).

\section{References}

1- Tonetti MS, Greenwell H, Kornman KS. Staging and grading of periodontitis: framework and proposal of a new classification and case definition. J Clin Periodontol. 2018;45(20):149-61. doi: 10.1002/ JPER.18-0006

2- Kassebaum NJ, Bernabé E, Dahiya M, Bhandari B, Murray CJ, Marcenes W. Global burden of severe periodontitis in 1990-2010: a systematic review and meta-regression. J Dent Res. 2014;93(11):104553. doi: 10.1177/0022034514552491

3- Dörfer C, Benz C, Aida J, Campard G. The relationship of oral health with general health and NCDs: a brief review. Int Dent J. 2017;67(2):14-18. doi: 10.1111/idj.12360

4- Portela A, Esteller M. Epigenetic modifications and human disease. Nat Biotechnol. 2010;28(10):1057-68. doi: 10.1038/nbt.1685 5- Weber M, Hellmann I, Stadler MB et al. Distribution, silencing potential and evolutionary impact of promoter DNA methylation in the human genome. Nat Genet. 2007;39(4):457-66. doi: 10.1038/ng1990 6- Fang JY, Xiao SD. Folic acid, polymorphism of methyl-group metabolism genes, and DNA methylation in relation to GI carcinogenesis. J Gastroenterol. 2003;38(9):821-9. doi: 10.1007/s00535-003-1207-7. 7- Nash AJ, Mandaviya PR, Dib MJ, Uitterlinden AG, van Meurs J, Heil $S G$, et al. Interaction between plasma homocysteine and the MTHFR c. $677 \mathrm{C}>\mathrm{T}$ polymorphism is associated with site-specific changes in DNA methylation in humans. Arch Oral Biol. 2016;72:66-74. doi: 10.1016/j. archoralbio.2016.08.014

8- Naghibalhossaini F, Mokarram P, Khalili E, Naghibalhossaini S. DNMT3b $-149 \mathrm{C} / \mathrm{T}$ promoter variants and methylation of colorectal cancer-associated genes. Cancer Biomark. 2015;15(3):227-33. doi: 10.3233/CBM-150463

9- Oliveira NF, Damm GR, Andia DC, Salmon C, Nociti FH Jr, Line SR, et al. DNA methylation status of the IL8 gene promoter in oral cells of smokers and non-smokers with chronic periodontitis. J Clin Periodontol. 2009;36(9):719-25. doi: 10.1111/j.1600-051X.2009.01446.x

10- Larsson L. Current concepts of epigenetics and its role in periodontitis. Curr Oral Health Rep. 2017;4(4):286-93. doi: 10.1007/ s40496-017-0156-9

11- Irwandi, RA, Vacharaksa A. The role of microRNA in periodontal tissue: a review of the literature. Arch Oral Biol. 2016;72:66-74. doi: 10.1016/j.archoralbio.2016.08.014

12- Wang $Y$, Andrukhov $O$, Rausch-Fan $X$. Oxidative stress and antioxidant system in periodontitis. Front Physiol. 2017;8:910. doi: $10.3389 /$ fphys.2017.00910
13- Liew SC, Gupta ED. Methylenetetrahydrofolate reductase (MTHFR) C677T polymorphism: epidemiology, metabolism and the associated diseases. Eur J Med Genet. 2015;58(1):1-10. doi: 10.1016/j. ejmg.2014.10.004

14- Santos Nunes MK, Silva AS, Queiroga Evangelista IW, Filho JM, Gomes CN, Nascimento RA, et al. Hypermethylation in the promoter of the MTHFR gene is associated with diabetic complications and biochemical indicators. Diabetol Metab Syndr. 2017;9:84. doi: 10.1186/ s13098-017-0284-3

15- Fonseca-Silva T, Oliveira MV, Fraga CA, Farias LC, Gomes EP, Barros LO, et al. DNMT3B (C46359T) polymorphisms and immunoexpression of DNMT3b and DNMT1 proteins in oral lichen planus. Pathobiology. 2012;79(1):18-23. doi: 10.1159/000330171

16- Ye P, Simonian M, Chapple CC, Gibbins JR, Kumar RK, Hunter $N$. Differential expression of transforming growth factors-beta 1, -beta 2, -beta 3 and the type I, II, III receptors in the lining epithelia of inflamed gingiva. Pathology. 2003;35(5):384-92. doi: 10.1080/00313020310001602585

17- Zhang J, Jia J, Zhao L, Li X, Xie Q, Chen X, et al. Down-regulation of microRNA-9 leads to activation of IL-6/Jak/STAT3 pathway through directly targeting IL-6 in HeLa cell. Mol Carcinog. 2016;55(5):732-42. doi: $10.1002 / \mathrm{mc} .22317$

18- Armitage GC. Development of a classification system for periodontal diseases and conditions. Ann Periodontol. 1999;4(1):1-6. doi: 10.1902/ annals.1999.4.1.1

19- Aidar M, Line SR. A simple and cost-effective protocol for DNA isolation from buccal epithelial cells. Braz Dent J. 2007;18(2):148-52. doi: 10.1590/s0103-64402007000200012

20- Arruda VR, Von Zuben PM, Chiparini LC, Annichino-Bizzacchi JM, Costa FF. The mutation Ala677-->val in the methylene tetrahydrofolate reductase gene: a risk factor for arterial disease and venous thrombosis. J Thomb Haemost. 1997;77:818-21. doi: 10.1590/S141547572013005000035

21- Farias LC, Fraga CA, Oliveira MV, Silva TF, Marques-Silva L, Moreira $P R$, et al. Effect of age on the association between p16CDKN2A methylation and DNMT3B polymorphism in head and neck carcinoma and patient survival. Int J Oncol. 2010;37(1):167-76. doi: 10.3892/ ijo_00000664

22- Costa LA, Silva IC, Mariz BA, Silva MB, Freitas-Ribeiro GM, Oliveira NF. Influence of smoking on methylation and hydroxymethylation levels in global DNA and specific sites of KRT14, KRT19, MIR-9-3 and MIR-137 genes of oral mucosa. Arch Oral Biol. 2016;72:56-65. doi: 10.1016/j. archoralbio.2016.08.013

23- Silva MB, Melo AR, Costa LA, Barroso H, Oliveira NF. Global and gene-specific DNA methylation and hydroxymethylation in human skin exposed and not exposed to sun radiation. An Bras Dermatol. 2017;92(6):793-800. doi: 10.1590/abd1806-4841.20175875

24- Xiong Z, Laird PW. COBRA: a sensitive and quantitative DNA methylation assay. Nucleic Acids Res. 1997;25(12):2532-4. doi: 10.1093/nar/25.12.2532

25- Toy VE, Uslu MO. Do genetic polymorphisms affect susceptibility to periodontal disease? A literature review. Niger J Clin Pract. 2019;22(4):445-53. doi: 10.4103/njcp.njcp_462_18

26- Shen $\mathrm{H}$, Wang $\mathrm{L}$, Spitz MR, Hong WK, Mao L, Wei Q. A novel polymorphism in human cytosine DNA-methyltransferase-3B promoter is associated with an increased risk of lung cancer. Cancer Res. 2002;62(17):4992-5.

27- Zhang XM, Li S, Zhang QM. DNA methyltransferase 3B -149C/T polymorphism and the risk of laryngeal squamous cell carcinoma: a case-control study. Genet Mol Res. 2015;14(4):12866-71. doi: 10.4238/2015.October.21.6

28- Palioto DB, Finoti LS, Kinane DF, Benakanakere M. Epigenetic and inflammatory events in experimental periodontitis following systemic microbial challenge. J Clin Periodontol. 2019;46(8):819-29. doi: $10.1111 /$ jcpe. 13151 . 
29- Ge W, Jiao Y, Chang L. The association between MTHFR gene polymorphisms (C677T, A1298C) and oral squamous cell carcinoma: a systematic review and meta-analysis. PLoS One. 2018;13(8):e0202959. doi: 10.1371/journal.pone.0202959

30- Rashed L, Abdel Hay R, AlKaffas M, Ali S, Kadry D, Abdallah S. Studying the association between methylenetetrahydrofolate reductase (MTHFR) 677 gene polymorphism, cardiovascular risk and lichen planus. J Oral Pathol Med. 2017;46(10):1023-9. doi: 10.1111/jop.12588 31- Bezerra SF, Costa LA, Freitas PA, Oliveira NF. Age-related changes in DNA methylation status of hTERT gene promoter of oral epithelial cells. Braz Arch Biol Technol. 2015;58(1):82-89. doi:1590/S15168913201400029 .

32- Marinho MC, Pacheco AB, Costa GC, Ortiz ND, Zajdenverg L, Sansone C. Quantitative gingival crevicular fluid proteome in type 2 diabetes mellitus and chronic periodontitis. Oral Dis. 2019;25(2):58895. doi: 10.1111/odi.12996

33- Zhang Q, Wang LQ, Wong KY, Li ZY, Chim CS. Infrequent DNA methylation of miR-9-1 and miR-9-3 in multiple myeloma. J Clin Pathol. 2015;68(7):557-61. doi: 10.1136/jclinpath-2014-202817

34- Braga EA, Loginov VI, Filippova EA, Burdennyi AM, Pronina IV, Kazubskaya TP, et al. Diagnostic value of a group of microRNA genes hypermethylated in ovarian carcinoma. Bull Exp Biol Med. 2018;166(2):253-6. doi: 10.1007/s10517-018-4326-0

35- Cardoso EM Reis C, Manzanares-Céspedes MC. Chronic periodontitis, inflammatory cytokines, and interrelationship with other chronic diseases. Postgrad Med. 2018;130(1):98-104. doi: $10.1080 / 00325481.2018 .1396876$
36- Milani P, Gagliardi S, Cova E, Cereda C. SOD1 transcriptional and posttranscriptional regulation and its potential implications in ALS. Neurol Res Int. 2011;2011:458427. https://doi.org/10.1155/2011/458427 37- Glorieux C, Zamocky M, Sandoval JM, Verrax J, Calderon PB. Regulation of catalase expression in healthy and cancerous cells. Free Radic Biol Med. 2015;87:84-97. doi: 10.1016/j. freeradbiomed.2015.06.017

38- Oates N, Pamphlett R. An epigenetic analysis of SOD1 and VEGF in ALS. Amyotroph Lateral Scler. 2007;8(2):83-6. doi: 10.1080/17482960601149160

39- Tóthová L, Celec P. Oxidative stress and antioxidants in the diagnosis and therapy of periodontitis. Front Physiol. 2017;8:1055. doi: 10.3389/fphys.2017.01055

40- Min JY, Lim SO, Jung G. Downregulation of catalase by reactive oxygen species via hypermethylation of CpG island II on the catalase promoter. FEBS Lett. 2010;584(11):2427-32. doi: 10.1016/j. febslet.2010.04.048

41- Chibber S, Sangeet A, Ansari SA. Downregulation of catalase by $\mathrm{CuO}$ nanoparticles via hypermethylation of $\mathrm{CpG}$ island II on the catalase promoter. Toxicol Res (Camb). 2017;6(3):305-11. doi: 10.1039/c6tx00416d

42- Punj A, Shenoy S, Kumari NS, Pampani P. Estimation of antioxidant levels in saliva and serum of chronic periodontitis patients with and without ischemic heart disease. Int J Dent. 2017;2017:1965697. doi: $10.1155 / 2017 / 1965697$ 